\title{
Gestión del riesgo operacional en la banca universal venezolana
}

\author{
Soto Quintana, Antonio José* \\ Stagg, Marcial** \\ Valente Martínez, María Rosa***
}

\begin{abstract}
Resumen
El objetivo de este artículo es realizar un diagnóstico de la gestión del riesgo operacional en la banca universal venezolana. La tipología de la presente investigación fue considerada como descriptiva, transversal y de campo, la muestra la cual contempló los seis primeros bancos universales (en términos de captaciones de depósitos) ubicados en el territorio nacional fue seleccionada a través de un muestreo no probabilístico intencional. La técnica de recolección de datos consistió en un cuestionario auto-administrado tipo escala Likert, el cual estuvo dirigido a los gerentes adscritos a las diferentes unidades de riesgos de las oficinas principales de las instituciones bancarias de tipo universal. Los resultados revelan que la banca universal no difunde la forma como administran el riesgo operacional. Igualmente a pesar de motivar la creación de modelos que permitan la medición del riesgo operacional, dichos esfuerzos se ven neutralizados, pues dichos modelos internos no son puestos en ejecución por parte de las instituciones. Finalmente el manejo de tecnologías constituyen un efecto diferenciador de la muestra encuestada, originando una segmentación del negocio bancario, lo cual a largo plazo, perjudica a las instituciones en su conjunto dado el efecto del riesgo sistémico.
\end{abstract}

Palabras clave: Riesgo operacional, banca universal, gestión, diagnóstico.

* $\quad$ Centro de Estadística e Investigación de Operaciones. Facultad de Ciencias Económicas y Sociales. Universidad del Zulia. Maracaibo-Venezuela. E-mail: ajsoto10@hotmail.com

** Centro de Estadística e Investigación de Operaciones. Facultad de Ciencias Económi casy Sociales. Universidad del Zulia. Maracaibo-Venezuela. E-mail: marcialstagg@gmail.com

*** Instituto de Investigaciones. Facultad de Ciencias Económicas y Sociales. Universidad del Zulia. Maracaibo-Venezuela. E-mail: marivalente72@hotmail.com 


\title{
Operational Risk Management in Venezuelan Universal Banking
}

\begin{abstract}
The objective of this article is to make a diagnosis of operational risk management in Venezuelan universal banking. This investigation was classified as descriptive, transversal, field research; the sample consisted of the first six universal banking institutions (in terms of deposit capture) located in the national territory, selected using a non-probabilistic, intentional sample. The data collecting technique consisted of a self-administered Likert-scale questionnaire applied to managers in the different risk units of the main offices for universal-type banking institutions. Results of this investigation show that universal banks do not give out information about how they administer operational risk. Likewise, despite motivation for creating models that permit measuring operational risk, the above mentioned efforts get neutralized because these internal models are not executed by the institutions. Finally, technological management constitutes a differentiating effect on the polled institutions; originating a segmentation of the banking business, which in the long term, harms the institutions as a whole due to the effect of systemic risk.
\end{abstract}

Key words: Operational risk, universal banking, management, diagnostic.

\section{Introducción}

El sistema financiero de un país y especialmente su sistema bancario constituyen el principal mecanismo de movilización de recursos entre los distintos sectores productivos de un país. Este rol protagónico tiene su basamento en la transformación del ahorro en inversión, situación ésta que contribuye a sustentar el crecimiento de una nación.

En este orden de ideas Galindo (2004) señala, el sistema financiero juega un papel central para estimular el crecimiento de una nación. Por una parte los sistemas financieros movilizan y agrupan ahorro, por otra, promueven una asignación eficiente del capital.

Según la posición del autor, los buenos sistemas financieros monitorean las inversiones y ejercen gobierno corporativo sobre las firmas, garantizando que los fondos prestados tengan su uso más eficiente. De igual forma los intermediarios financieros también transforman, diversifican y manejan diversos riesgos de una manera que no podrían hacerlo los ahorristas individuales.

Para Bercoff (1999) y Moreno (2004), la importancia fundamental del sistema bancario radica en el enorme grado de influencia que éste ejerce sobre otros sectores de la economía. De hecho, su deterioro provoca entre otras cosas la interrupción del flujo del crédito a las familias y empresas, reducción de la inversión y el consumo y en algunos casos, encamina a la quiebra a empresas.

De allí que una deficiente administración bancaria ocasiona no sólo la quiebra o intervención de la entidad por parte de los entes gubernamentales, sino que puede en algunos casos generar un efecto negativo sobre la integridad de otras instituciones 
Gestión del riesgo operacional en la banca universal venezolana

Soto Quintana, Antonio José; Stagg, Marcial y Valente Martínez, María Rosa

bancarias provocando crisis sistémicas lo que se traducen en importantes desequilibrios para economía de un país en su conjunto.

Dada esta situación, es menester contar con un sistema bancario eficiente, competitivo y transparente, que logre una óptima asignación de sus recursos, permitiendo por un lado maximizar sus utilidades y por otro coadyuvando al crecimiento económico y social de la nación.

Sin embargo, la anterior declaración no siempre es tan fácil de llevar a la práctica, principalmente cuando el sistema bancario desarrolla sus actividades en mercados complejos que lo obligan a actuar en espacios inestables en los cuales una adecuada administración de los riesgos es en sí, una vía para alcanzar los objetivos estratégicos de la institución.

De allí que el Comité de Basilea (2003), Feria y Samaniego (2004) aseveren, la desregulación y globalización de los servicios financieros, junto con la creciente sofisticación de los sistemas tecnológicos financieros, están incrementando la complejidad de las actividades de los bancos y por ende, sus perfiles de riesgo.

Tales aseveraciones concuerdan con las expresadas por Cardona (2004) quien afirma, la actividad de una entidad financiera por esencia es la toma de riesgos, cada una de sus operaciones contiene implícita o explícitamente la incertidumbre. Las distintas dimensiones de su operación están expuestas a diferentes tipos de riesgo lo cuales deben ser identificados, medidos y controlados, como base para fijar las estrategias de mercadeo y de precios, que resulten en una ecuación favorable entre el riesgo asumi- do y la recompensa obtenida, medida como la rentabilidad neta del negocio.

En consecuencia dada la presencia del riesgo en el sistema bancario, su correcta administración contribuye sin duda alguna a la creación de valor para la empresa en el tiempo y constituye un elemento diferenciador que forma parte de una ventaja competitiva basada en la administración de los riesgos inherentes a la actividad bancaria.

Por tal motivo, el presente artículo está orientado a la realización de un diagnóstico de la gestión de uno de los principales riesgos que enfrentan las instituciones financieras como lo es el riesgo operacional, el cual a juicio de Nieto (2005) constituye un elemento de suma importancia para el sano desenvolvimiento del más importante subsistema del financiero venezolano como lo es la banca universal.

Para tal fin, la investigación fue considerada como transversal-descriptiva planteando un enfoque cuantitativo pues pretende ponderar los resultados obtenidos de la aplicación de un cuestionario autoadministrado tipo escala Likert, el cual estuvo dirigido a los gerentes adscritos a las diferentes unidades de riesgos de las oficinas principales de las instituciones bancarias de tipo universal ubicadas en el territorio nacional.

La población estuvo constituida por las entidades financieras de tipo universal que se encuentran ubicadas dentro del territorio venezolano y cuyos informantes fueron los gerentes de riesgo de las respectivas organizaciones.

En relación a la muestra, la misma fue seleccionada a través de un muestreo no probabilístico opinático o intencional, 
en el cual se eligieron las seis (6) primeras instituciones bancarias de tipo universal en términos de captaciones de depósitos; información ésta que se recogió de los informes emitidos por la Superintendencia de bancos y otras instituciones financieras.

Dentro de las instituciones seleccionadas que conformaron el estudio se encuentran: Banesco, Venezuela (BSCH), Mercantil, BBVA Provincial, Occidental de Descuento y Banfoandes, las cuales representan más del $58,72 \%$ del total de depósitos de la banca universal venezolana.

\section{Consideraciones teóricas sobre el riesgo operativo en el sistema bancario}

La necesidad de los sistemas financieros en enfrentar los retos contemporáneos caracterizados por la globalización, la competitividad y un marcado ritmo de aceleración en el ámbito tecnológi$c o$, exige al sector bancario adaptarse a los nuevos paradigmas; el cual concibe a la variable riesgo como el principal elemento a tomar en cuenta en las decisiones de inversión.

De esta forma, en el presente punto se presentará una visión integral del riesgo para luego puntualizar el concepto de riego operativo u operacional y como éste es abordado por las principales instituciones bancarias que realizan sus actividades dentro del territorio nacional

De acuerdo a Cade (1999), el riesgo es un terreno donde concurren lingüistas, filósofos, matemáticos, actuarios... y no hay definición única que sirva a todos ellos. Sin embargo, Markowitz (1959), Mao (1970), March y Shapira (1987) cita- do por Grifell y Marqués (2003), el riesgo se asocia al fracaso (resultados negativos) en conseguir el resultado objetivo o la posibilidad de no llegar a los resultados planeados, definición esta que concuerda con lo planteado por Aaker y Jacobson (1987) quienes consideran el riesgo como la probabilidad de pérdida o de la no consecución de una cierta rentabilidad objetivo.

Paralelamente, Grifell y Marqués (2003) señalan, la definición común de riesgo permite enfocarlo desde una perspectiva ex ante y una en el contexto ex post, la primera de ellas es la exposición a la posibilidad de la no consecución de los objetivos previstos para la actividad, mientras que la segunda representa el grado en que no se han conseguido los objetivos previstos para la actividad.

\subsection{El riesgo en la esfera empresarial}

Para García et al., (1998), dado que la definición de riesgo es muy amplia y puede aplicarse a muy diversos aspectos y situaciones, tanto en el ámbito empresarial como fuera del mismo; el autor visualiza al riesgo como una situación en la que no existe garantía de éxito seguro, lo que en términos económicos va íntimamente relacionado con la posibilidad de obtener un lucro.

Según Ponce y Villanueva (1998), los empresarios definen el riesgo como amenazas que se originan por circunstancias que pueden afectar adversamente la habilidad de la organización para lograr sus objetivos y ejecutar sus estrategias satisfactoriamente, es decir, es el conjunto de circunstancias que pueden disminuir el beneficio empresarial. 
Gestión del riesgo operacional en la banca universal venezolana

Soto Quintana, Antonio José; Stagg, Marcial y Valente Martínez, María Rosa

Los riesgos a que está expuesta una empresa son diversos e influyen en todas las decisiones de la organización, por consiguiente puede haber distintos factores que afecten el resultado del ejercicio económico de la empresa.

De acuerdo al autor, una primera clasificación de riesgos empresariales puede ser como la que sigue a continuación:

1. El riesgo económico: Es aquél que se asocia con las operaciones normales de la empresa o más precisamente, es el riesgo de no poder cubrir los costos de operación. Es independiente de la estructura financiera de la empresa, e incluye aquellas eventualidades que pueden afectar el resultado de explotación de la empresa, tales como obsolescencia o exceso de oferta del producto, baja de cotizaciones en mercados, entre otras. Estas se derivan de la incapacidad de la organización para garantizar la estabilidad del resultado, debido a que se encuentra expuesta a factores endógenos y exógenos propios de su entorno.

Los dos componentes del riesgo económico son el peligro que la empresa fracase, debido a la incapacidad de los activos para generar un nivel suficiente de utilidades antes de intereses e impuestos, y la variabilidad de tales utilidades.

2. El riesgo financiero: Se crea por el establecimiento de préstamos y acciones preferentes; está altamente correlacionado con la estructura financiera de la empresa y se refiere a cuando no se puede cubrir las obligaciones.

Los dos componentes del riesgo financiero son el peligro que la empresa fracase por incapacidad de cubrir los pagos por intereses y/o capital de la deuda $y$, la variabilidad de las utilidades disponibles para las acciones comunes que se causa por los cargos financieros fijos.

3. El riesgo diversificable: Es aquél que disminuye cuando se invierte en más de un activo. A este tipo de clasificación de riesgo también se le denomina no sistemático o único. Existen activos cuya rentabilidad en el tiempo va en la misma dirección, y otros cuya rentabilidad va en sentido contrario.

4. El riesgo no diversificable: Es aquél que no puede reducirse, aún cuando se invierta en varios activos debido a que afecta a todos los sectores en general. A este tipo de clasificación de riesgos también se les llama sistemático o de mercado.

De las clasificaciones anteriores de riesgo, el autor señala que una empresa está en capacidad de obtener una agrupación distinta con características comunes en cuanto a su medición, éstas se pueden referir a riesgos cuantificables y no cuantificables.

Bessis (2002), avanza en la definición del riesgo desde una perspectiva bancaria, al conceptualizarlo como el impacto adverso en el rendimiento, debido a diferentes fuentes de incertidumbre.

De acuerdo a García et al (1998), el riesgo es un elemento inherente de la actividad bancaria y afecta tanto a los bancos privados como a los bancos centrales. Al igual que cualquier actividad empresarial, por el simple hecho de encontrarse en un entorno de incertidumbre, la actividad bancaria se ve sometida a una serie de riesgos, motivados precisamente por la falta de certeza sobre el futuro de sus operaciones y de la organización. 
Paralelamente, Guillén (2002), García et al (1998) señalan, las crisis bancarias surgen debido a la elevada exposición que poseen las instituciones bancarias a riesgos muy diversos y al inadecuado manejo del mismo. Dichos riesgos surgen ya que estas instituciones fungen de intermediarios entre agentes económicos poseedores de flujos financieros excedentes o de ahorros, y agentes económicos deficitarios que demandan fondos de créditos para costear sus inversiones o niveles de consumo.

Al fungir de intermediarios entre agentes económicos demandantes y oferentes de recursos financieros o fondos prestables, las instituciones bancarias adquieren activos y asumen pasivos que pueden presentar asimetrías de diversa índole, lo que en determinadas circunstancias puede afectar su viabilidad financiera o solvencia económica como empresa.

Por otra parte Padoa-Schioppa (1998) señalan, la globalización entendida en sus dos vertientes: globalización geográfica (supresión de las fronteras financieras entre naciones) y globalización funcional (supresión de la frontera que diferenciaba los instrumentos financieros de los servicios financieros, debido a la aparición de nuevos productos que hace difícil su inclusión en uno de los dos grupos) ha originado tanto la presencia de entidades financieras en mercados financieros diferentes a los tradicionales, como la utilización de nuevos productos financieros; situación ésta que ha coadyuvado a la aparición de nuevos riesgos y la intensificación de los riesgos bancarios clásicos.

Según Sebastián y López (2001), dada su propia naturaleza, los bancos han asumido desde siempre distintos ti- pos de riesgo, sin embargo, lo que a primera vista podría parecer un viejo y conocido problema presenta nuevos matices debido a que los beneficios ya no son los de antes y el capital debe adecuarse al riesgo.

En opinión de los autores, la inestabilidad de los mercados, la tendencia de los bancos a aumentar el peso de su cartera de valores y la utilización de los productos derivados, hace que las instituciones sean más sensibles a la volatilidad de los mercados financieros.

De acuerdo a Pérez y Quesada (1992), los bancos asumen riesgos como consecuencia de un entorno más incierto, de allí que los costes asociados a la cobertura de los mismos no se puede atribuir a ineficiencias de las empresas bancarias, sino de la economía en la que actúan.

De allí que Ortiz (2005) señale, es innegable que el modelo gerencial alimentado por la gestión de riesgos empresariales parte de la identificación y clasificación de los riesgos y señala que aunque no todos los riesgos inciden en los objetivos y las políticas gerenciales, es pertinente disponer de una descripción global de los mismos y señala que la lista universal de riesgos y de sus características constituyen el marco de referencia para precisar cuáles de ellos merecerán la evaluación interna cuidadosa y la posterior selección de las opciones encaminadas a reducir o eliminar sus repercusiones negativas.

\subsection{El riesgo operativo y la banca universal venezolana}

De acuerdo a los planteamientos de Nieto (2005), en los últimos años se ha observado un crecimiento progresivo de la preocupación de las entidades finan- 
Gestión del riesgo operacional en la banca universal venezolana

Soto Quintana, Antonio José; Stagg, Marcial y Valente Martínez, María Rosa

cieras por el riesgo operacional, el cual siempre ha existido en la banca, pero que quizás ahora se manifiesta con mayor intensidad, debido a múltiples factores tales como las mejoras experimentadas en las tecnologías, dependencia en procesos automáticos, la bancarización, el desarrollo del comercio electrónico, la creciente complejidad y globalización del sistema financiero.

Este riesgo operacional es definido por Freixas y Saurina (2004) como la posibilidad de pérdidas por falta de adecuación o fallos en los procesos, personas, sistemas internos, o bien como consecuencia de acontecimientos externos.

Tal definición es compartida por PriceWatersHouseCoopers (2003) quien distingue cuatro categorías de riesgo dentro del riesgo operacional, a saber:

1. Personal. Pérdidas asociadas con violaciones intencionales de las políticas internas por parte del personal actual o que ya no labora en la empresas.

2. Procesos. Pérdidas que han sido incurridas por deficiencias en algún procedimiento vigente, o por la falta de algún procedimiento. Las pérdidas en esta categoría pueden derivarse de errores o fallas del personal en el seguimiento de algún procedimiento.

3. Sistemas. Pérdidas ocasionadas por fallas en los sistemas o la tecnología actuales.

4. Externos. Pérdidas como resultado de fuerzas de la naturaleza o como resultado de eventos o situaciones ocasionados por terceros.

En opinión de Sebastián y López (2001), el riesgo operacional está relacionado en la mayoría de los casos con fallos ocurridos en el proceso de segui- miento y control de las posiciones asumidas; y por lo tanto, muchas de las quiebras bancarias se atribuyen según los autores antes citados a un inadecuado fallo del control interno. En este sentido, el problema puede deberse a un fallo en la parte de gestión que fue incapaz de supervisar adecuadamente a los empleados que exponían el banco a pérdidas, o simplemente es evidencia de políticas mal definidas las cuales condujeron a los bancos inadvertidamente a la quiebra.

De allí que Cea (2002) señale, aunque el riesgo operacional es inherente a todas las operaciones de negocios y no puede ser eliminado totalmente, si puede ser gestionado, mitigado $\mathrm{y}$, en algunos casos, asegurado.

En tal sentido, el Banco Internacional de Pagos (2003) señala una clasificación detallada de cómo identificar el riesgo operacional a través de distintos tipos de eventos de pérdida, englobando tres niveles.

El primer nivel enumera siete tipos de eventos que tienen la consideración de pérdida por riesgo operacional entre los que se consideran: Fraude interno (actos internos), fraude externo (actos externos), relaciones laborales y seguridad en el puesto de trabajo, prácticas con clientes, productos y negocios, daños a activos materiales e incidencias en el negocio, fallos en los sistemas y la ejecución, entrega y gestión de procesos.

Existe además un segundo nivel, que aporta un desglose superior al anterior, y un tercer nivel, que añade ejemplos de actividades de cada una de las categorías del nivel 2.

Por otro lado, Marroquín (2003) señala que como posibles causas del riesgo 
operativo se encuentran la globalización, crecimiento de los medios electrónicos, fusiones y adquisiciones a gran escala, mayor automatización tecnológica, mayor oferta de proveedores de servicios, incremento del outsourcing, complejidad y cobertura de productos, crecimiento de volúmenes de negocio y las regulaciones y normativas entre otros.

Igualmente plantea que según cifras del Banco Internacional de Pagos, la categoría de riesgo operativo más frecuente la constituye el fraude externo seguido de la ejecución, entrega y gestión de procesos (Tabla 1).

En tal sentido, la autora antes mencionada señala la necesidad de llevar a cabo un proceso de revisión y supervisión tal como lo contempla el segundo pilar de Basilea, en el cual los bancos deben divulgar información suficiente para permitir a los participantes del mercado evaluar su exposición al riesgo operativo y la calidad de su gestión de riesgo. Igualmente, los bancos podrán tener un perfil de ries- go el cual podría ser revisado tanto por sus clientes como por sus accionistas, así como un índice de competitividad.

La situación antes señalada origina la necesidad de crear una unidad independiente responsable del riesgo operativo que cumpla con funciones de identificación, medición, monitoreo y control, así como el diseño e implantación de metodologías de gestión para el riesgo operativo.

Por su parte el Banco Internacional de Pagos (2003), recoge diez prácticas o principios para un adecuado control y administración del riesgo operacional los cuales se logran a través del desarrollo de una cultura adecuada de riesgo, la gestión de riesgos, el papel de los supervisores y la divulgación.

En cuanto a la cultura de riesgo se han desarrollado los siguientes principios:

Principio 1: El Consejo de Administración ha de ser consciente de los principales aspectos de los riesgos operacionales del banco.

Tabla 1

Distribución de pérdidas por tipo de evento

\begin{tabular}{lcc}
\hline \multicolumn{1}{c}{ Tipo de evento } & Tipo de evento severidad (\%) & Frecuencia (\%) \\
\hline Ejecución, entrega y gestión de procesos & 29 & 35 \\
Interrupción de operaciones y fallos de sistemas & 3 & 1 \\
Daños a activos físicos & 24 & 1 \\
Clientes, productos y prácticas comerciales & 13 & 7 \\
Prácticas de empleo y seguridad laboral & 7 & 9 \\
Fraude externo & 16 & 42 \\
Fraude interno & 7 & 3 \\
Otros & 1 & 1 \\
\hline
\end{tabular}

Fuente: Banco Internacional de Pagos (2004). 
Gestión del riesgo operacional en la banca universal venezolana

Soto Quintana, Antonio José; Stagg, Marcial y Valente Martínez, María Rosa

Principio 2: El Consejo de Administración debe asegurar que el esquema de gestión del riesgo operacional del banco esté sujeto a una auditoria interna efectiva e integral.

Principio 3: La alta dirección de la compañía debe tener la responsabilidad de implementar el esquema de gestión del riesgo operacional aprobado por el Consejo de Administración.

Con respecto a la Gestión de riesgos (que involucra identificación, evaluación, seguimiento, control y mitigación de riesgos) se esbozan a continuación cuatro principios:

Principio 4: Los bancos deben identificar y evaluar el riesgo operacional inherente a todos los productos, actividades, procesos y sistemas relevantes.

Principio 5: Los bancos deben implementar un proceso para el seguimiento regular de los perfiles del riesgo operacional y de su exposición material a pérdidas.

Principio 6: Los bancos deben tener políticas, procesos y procedimientos para controlar o mitigar los riesgos operacionales más significativos.

Principio 7: Los bancos deben implementar planes de contingencia y de continuidad del negocio a fin de garantizar su capacidad para operar en forma continua y minimizar las pérdidas en caso de una interrupción severa del negocio.

En relación al papel de los supervisores se plantean:

Principio 8: Los supervisores deben exigir a todos los bancos, sin importar su tamaño, que implementen un esquema eficaz para identificar, evaluar, seguir y controlar o mitigar los riesgos operacionales materiales como parte de un enfoque integral para la gestión de riesgos.
Principio 9: Los supervisores deben llevar a cabo, de manera directa o indirecta, una evaluación periódica independiente de las políticas, procedimientos y prácticas de un banco relacionadas con el riesgo operacional.

$Y$ en cuanto al papel de la divulgación, el principio 10 establece que los bancos deben realizar suficiente divulgación pública para permitir que los participantes del mercado evalúen su enfoque para la gestión del riesgo operacional.

Finalmente, es importante destacar que Jiménez y Martín (2005; citado por Nieto, 2005), al evaluar las limitantes presentadas por la gestión del riesgo operacional señalan la carencia de bases de datos internas para aproximar las variables a utilizar en el modelo. Por ello, el Acuerdo de Basilea II propone la complementariedad de estos datos con bases externas, las cuales agreguen información sobre sucesos, principalmente de baja frecuencia y media o alta severidad, que posiblemente la entidad no haya experimentado, pero a los que sí está expuesta.

\section{Análisis de la evaluación del riesgo organizacional}

A continuación se presentan los resultados obtenidos de la aplicación del instrumento de investigación a los once (11) gerentes adscritos a las diversas unidades de riesgo que operan en la banca universal venezolana, los cuales son expresados en frecuencias absolutas y relativas y representadas en una tabla sinóptica, con el correspondiente análisis descriptivo de dichos resultados. 
Con respecto a la evaluación del riesgo operacional mostrado en la Tabla 2 se aprecia que solamente el $36 \%$ de las instituciones realiza una asignación de los roles para el cálculo del riesgo operacional lo cual representa una brecha significativa en las distintas instituciones que componen subsistema del sistema financiero venezolano como lo es la banca universal.

Por otra parte, a pesar que el $82 \%$ de los gerentes de riesgo encuestados reconocen y desarrollan una cultura integral del riesgo operacional y ésta es percibida como una ventaja competitiva y no una mera disposición de carácter regulatorio, sólo el $54 \%$ de las instituciones capacita a sus empleados para el control y mitigación del riesgo operacional.

En lo relacionado a la divulgación de resultados, sólo un $27 \%$ de las instituciones realiza la divulgación pública y abierta de su enfoque para la gestión del riesgo lo que evidencia la necesidad de crear un mayor nivel de concienciación de la importancia que estas publicaciones poseen.

Tabla 2

Evaluación del riesgo operacional

\begin{tabular}{|c|c|c|c|c|}
\hline \multirow[t]{2}{*}{ Items } & \multicolumn{2}{|c|}{ Presencia } & \multicolumn{2}{|c|}{ Ausencia } \\
\hline & FA & FR & FA & FR \\
\hline $\begin{array}{l}\text { 1) La institución realiza una asignación de } \\
\text { roles para el cálculo del riesgo operacional. }\end{array}$ & 4 & $36 \%$ & 7 & $64 \%$ \\
\hline $\begin{array}{l}\text { 2) El banco desarrolla una cultura integral } \\
\text { del riesgo operacional que garantiza la } \\
\text { creación de valor para la empresa y no un } \\
\text { mero requisito de entes externos. }\end{array}$ & 9 & $82 \%$ & 2 & $18 \%$ \\
\hline $\begin{array}{l}\text { 3) La institución considera el adiestramiento } \\
\text { del personal para la evaluación y control del } \\
\text { riesgo operacional. }\end{array}$ & 6 & $54 \%$ & 5 & $45 \%$ \\
\hline $\begin{array}{l}\text { 4) El banco realiza la divulgación pública y } \\
\text { abierta de su enfoque para la gestión del } \\
\text { riesgo operacional. }\end{array}$ & 3 & $27 \%$ & 8 & $73 \%$ \\
\hline $\begin{array}{l}\text { 5) La organización evalúa el riesgo } \\
\text { operacional inherente a todas las } \\
\text { actividades del mismo. }\end{array}$ & 7 & $64 \%$ & 4 & $36 \%$ \\
\hline $\begin{array}{l}\text { 6) El banco considera que las alianzas } \\
\text { estratégicas son una vía de adquirir nuevas } \\
\text { tecnologías para facilitar la administración } \\
\text { del riesgo operacional. }\end{array}$ & 4 & $36 \%$ & 7 & $64 \%$ \\
\hline $\begin{array}{l}\text { 7) El banco propicia la creación de modelos } \\
\text { de medición de riesgos entre las distintas } \\
\text { unidades de riesgos. }\end{array}$ & 9 & $82 \%$ & 2 & $18 \%$ \\
\hline $\begin{array}{l}\text { 8) La organización implanta herramientas } \\
\text { avanzadas de apoyo a la medición de los } \\
\text { riesgos elaboradas por sus empleados. }\end{array}$ & 3 & $27 \%$ & 8 & $73 \%$ \\
\hline
\end{tabular}


Gestión del riesgo operacional en la banca universal venezolana

Soto Quintana, Antonio José; Stagg, Marcial y Valente Martínez, María Rosa

Los referidos hallazgos expresan de manera clara la poca transparencia del sistema bancario en cuanto a la administración de los riesgos, pues discrepan de lo expresado por Marroquin (2003), quien señala que dentro del segundo pilar de Basilea se contempla un proceso de revisión y supervisión, en el cual los bancos deben divulgar información suficiente que permita que los participantes del mercado evalúen su exposición al riesgo operativo y la calidad de su gestión de riesgo. Igualmente los bancos deben tener tanto un perfil de riesgo el cual podría ser revisado por sus clientes y/o accionistas, como un índice de competitividad.

Por otra parte, existe un porcentaje significativo de instituciones (36\%) las cuales no evalúan el riesgo operacional inherente a todas las actividades del mismo, situación que aunada a la no publicación de tal evaluación por parte de las instituciones que sí lo llevan a cabo; evidencia la falta de cultura en materia de este tipo de riesgo.

Por otra parte, se observa un bajo porcentaje de aceptación (36\%) sobre la posibilidad de creación de alianzas estratégicas como vía de adquirir nuevas tecnologías que coadyuven a un sano control de administración del riesgo en el sector bancario.

Estos resultados contrastan con los encontrados por Larrán y Muriel (2004) quienes señalan que, los grandes grupos financieros españoles han apostado por una estrategia de desarrollo interno de sus respectivas tecnologías, con el fin de aumentar el control real o percibido de dicho proceso de desarroIlo. Los autores aseveran que la tenden- cia al avance de la tecnología interna superaba el $40 \%$ del gasto total, lo que reflejaba el deseo de muchas entidades de mantener el control sobre el avance de sus aplicaciones.

Esta situación planteada por los grupos financieros europeos tiene su sustento en la creencia generalizada que la subcontratación reduce el control real o percibido sobre la actividad subcontratada, al ser desarrollada por personal ajeno a la entidad.

Adicionalmente, los gerentes de riesgo de las instituciones encuestadas demuestran la importancia que tiene el manejo de las tecnologías en la banca universal venezolana, de allí que el $82 \%$ de los encuestados aseguró que su institución propicia la creación de modelos de medición de riesgos entre las distintas unidades de riesgos. Sin embargo, sólo el $27 \%$ implanta herramientas avanzadas de apoyo a la medición de los riesgos.

Es importante destacar que la situación antes señalada evidencia una inconsistencia entre los insumos solicitados por la institución en materia de tecnologías, y aquellos que realmente logra poner en práctica; lo cual puede tornarse en el largo plazo en acciones que restrinjan acciones proactivas en materia de generación de modelos de riesgo.

De allí que Urcola (2000) al referirse a los principios que fomentan la innovación y la creatividad señala, las buenas ideas deben presupuestarse, programarse e implantarse, con el firme propósito de motivar a cada uno de los actores involucrados a continuar diseñando y ejecutar métodos más innovadores que faciliten la optimización de los recursos. 


\section{Consideraciones finales}

Partiendo de los procesos de transformación bancarios experimentados hoy en día a nivel mundial y específicamente los cambios ocurridos en la América Latina producto de crisis bancarias, la globalización financiera y el adelanto de las nuevas tecnologías, el manejo de la variable riesgo a constituido un elemento diferenciador entre las instituciones que han logrado un crecimiento económico estable de aquellas que no lo han tenido.

En este sentido, resulta incuestionable la importancia derivada de una administración del riesgo la cual contemple todas las aristas operativas del la institución bancaria, desde el simple monitoreo de las colocaciones en papeles comerciales hasta la percepción que posea los usuarios sobre la manera como son manejados los recursos que estos últimos les confieren a la institución.

De igual manera, producto del análisis de la gestión del riesgo operacional en la banca universal se plantean ciertos lineamientos estratégicos presentados a continuación, que coadyuven al manejo del riesgo operacional como curso de acción financiera en la banca universal venezolana.

El primer lineamiento estratégico considera que, la banca universal venezolana debe no sólo establecer sino difundir los avances que la institución posee en materia de riesgo operacional.

Las estructuras bancarias deben aportar la transparencia y los mecanismos necesarios para que la gestión de los riesgos de la entidad fluyan de forma ágil entre todos los actores involucrados dentro del sistema.
Igualmente, las entidades deben publicar en un período de tiempo razonable aquella información (tanto cuantitativa como cualitativa) que posibilite a los participantes en los mercados financieros realizar dichas evaluaciones, información que no sólo ha de ser fiable, sino también relevante y estar asentada en principios de valoración generalmente aceptados.

En definitiva, el curso de acción para mejorar el riesgo operacional es la promoción de una mayor transparencia que facilite la disciplina y supervisión de los mercados, permitiendo que el público y los supervisores evalúen mejor los perfiles de riesgo de las instituciones financieras, lo cual, en el mediano plazo coadyuvará a mejorar la reputación individual de la entidad financiera y del conjunto del sector.

El segundo lineamiento afirma que la banca universal debe incentivar la creación de modelos de gestión de riesgo bancarios a través del propio recurso humano.

Las organizaciones reconocen que uno de los activos más valiosos con que cuentan es su capital humano, en ellos se encuentra la capacidad de la organización de generar créditos sustentables en el tiempo, y a la vez permite fijar una diferenciación competitiva explicita de las otras organizaciones del ramo.

Este sentido, dado el profundo cambio de conciencia sobre la importancia de los medios técnicos y humanos necesarios para identificar los riesgos, los empleados deben estar conscientes de que su trabajo será no sólo evaluado sino puesto en ejecución, con el fin de generar una mayor motivación para todo creador de una idea al aceptarle sus sugerencias 
Gestión del riesgo operacional en la banca universal venezolana

Soto Quintana, Antonio José; Stagg, Marcial y Valente Martínez, María Rosa

y las ideas concebidas para la mitigación

y control de los riesgos.

Y por último, el tercer lineamiento considera que el manejo del riesgo operacional debe dejar de ser un elemento diferenciador en la banca universal venezolana.

El manejo de riesgo operacional constituyó un elemento diferenciador en la población encuestada además de presentar un estadio de desarrollo incipiente, ya que sólo el $64 \%$ de las instituciones bancarias evalúa el riesgo operacional inherente a todas las actividades del mismo. Dada la interdependencia de las instituciones financieras, tal situación es contraproducente en el largo plazo, los potenciales problemas que posee un banco puede generar un cambio en las expectativas de los agentes económicos y generar las corridas de bancos que se encuentren solventes financieramente.

\section{Referencias Bibliográficas}

Aaker David, Jacobson Robert (1987). The role of risk in explaining differences in profitability. Academy of Management Journal 30 (2).

Banco Internacional de Pagos (2003). Sound Practices for the Management and Supervision of Operational Risk. http://www.bis.org/publ/bcbs96.pdf. En línea [23/01/2005].

Banco Internacional de Pagos (2004). Comité de supervisión bancaria de Basilea. Gestión consolidada del riesgo KYC. http://www.bis.org/publ. [23/01/2005].

Bercoff, José (1999). Indicadores tempranos de debilitamiento del sector financiero argentino. Instituto de Investigaciones Económicas. Universidad Nacional de Tucumán.
Bessis, Joel (2002). Risk Management in Banking. John Wiley \& Sons, Ltd.

Cade, Eddie (1999). Managing Banking Risks, Fitzroy Dearbom Publishers.

Cardona, Paola (2004). Aplicación de árboles de decisión en modelos de riesgo crediticio. Revista Colombiana de Estadística. Volumen 27. No. 2.

Cea, José Manuel (2002). "Mitigación del Riesgo Operacional". II Jornadas de Riesgos Financieros. Madrid.

Comité de Basilea (2003). Sound Practices for the Management and Supervision of Operational Risk. http: //www.bis.org/publ/bcbs96.pdf. En línea [23/01/2005].

Feria, José y Samaniego Ruiz, Joseph (2004). La Banca en Internet: Riesgos Implícitos. http://thales.cica.es/rd/Recursos/rd98/Economia. En línea [23/01/2005].

Freixas, Xavier; Saurina, Jesús (2004). Teoría y práctica de la regulación bancaria. Moneda y crédito. Segunda época. № 218.

Galindo, Arturo (2004). El Papel del Sistema Bancario en el Crecimiento. Notas para el Coloquio Latino Americano. www.ycsg.yale.edu. En línea [08/04/2005].

García, Gustavo; Rodríguez, Rafael; Salvato, Silvia (1998). Lecciones de la Crisis Bancaria de Venezuela. Ediciones IESA. Caracas, Venezuela.

Grifell, Emilie; Marqués, Pilar (2003). El riesgo en dirección estratégica: concepto y medidas. http://cdiserver.mbasil.edu.pe. En línea [08/04/2005].

Guillén, Ruth (2002). Consideraciones preliminares de la gestión del riesgo en el sistema bancario-el caso de las crisis bancarias en Venezuela. http://webdelprofesor.ula.ve. En línea [23/01/2005]. 
Larrán, Manuel; Muriel, María (2004). El impacto de las nuevas tecnologías de la información en el negocio bancario español. Revista de empresa $\mathrm{n} 09$.

Marroquin, Yuri (2003). El riesgo operativo. http://www.clm.com.br. En línea [27/01/2005].

Moreno, Elena (2004). Crisis financieras: antecedentes teóricos y su relación con las crisis bancarias. http://www.eumed.net. En línea [23/01/2005].

Nieto, M. ․ Ángeles (2005). El tratamiento del riesgo operacional en Basilea II. http://dialnet.unirioja.es/servlet/oaiart?codigo $=1159114$. [23/01/2005]

Ortiz, Alberto (2005). Gerencia financiera y diagnóstico estratégico. Mc Graw Hill Interamericana, S.A. Segunda edición. Bogota Colombia. pp. 482.

Padoa-Schioppa, Tommaso (1998). Global Supervision: a Term in Search of a Content, Banca Nazionale de Lavoro Quarterly Review.
Pérez, Francisco; Quesada, Javier (1992). La eficacia del sistema bancario español en la perspectiva europea Revista Moneda y Banca 195.

Ponce, José; Villanueva, Juan (1998). Evaluación de riesgos IV Reunión de auditores internos de Banca Central. Centro de Estudios Monetarios Latinoamericanos - CEMLA. Cartagena de Indias, Colombia.

Price Waters House Coopers (2003). La Administración del Riesgo Operacional. http://www.pwcglobal.com. En línea [27/01/2005].

Sebastián González; A.; López Pascual, J. (2001). Gestión Bancaria. Los nuevos retos en un entorno global. Mc Graw Hill/Interamericana de España, S.A.U.

Urcola, Juan Luis (2000). Factores claves de dirección orientados a la obtención de resultados. Escuela Superior de Gestión Comercial y Marketing. Madrid-España. 